\title{
Seroprevalence and risk factors for influenza a viruses in pigs in Peninsular Malaysia.
}

\begin{abstract}
Following a series of H5N1 cases in chickens and birds in a few states in Malaysia, there was much interest in the influenza A viruses subtypes that circulate among the local pig populations. Pigs may act as a mixing vessel for avian and mammal influenza viruses, resulting in new reassorted viruses. This study investigated the presence of antibodies against influenza $\mathrm{H} 1 \mathrm{~N} 1$ and $\mathrm{H} 3 \mathrm{~N} 2$ viruses in pigs from Peninsular Malaysia using Herdcheck Swine Influenza H1N1 and H3N2 Antibody Test Kits. At the same time, the presence of influenza virus was examined from the nasal swabs of seropositive pigs by virus isolation and real time RT-PCR. The list of pig farms was obtained from the headquarters of the Department of Veterinary Services, Malaysia, and pig herds were selected randomly from six of 11 states in Peninsular Malaysia. A total of 727 serum and nasal swab samples were collected from 4- to 6-month-old pigs between May and August 2005. By ELISA, the seroprevalences of swine influenza $\mathrm{H} 1 \mathrm{~N} 1$ and $\mathrm{H} 3 \mathrm{~N} 2$ among pigs were $12.2 \%$ and $12.1 \%$ respectively. Seropositivity for either of the virus subtypes was detected in less than half of the 41 sampled farms $(41.4 \%)$. Combination of both subtypes was detected in $4 \%$ of all pigs and in $22 \%$ of sampled farms. However, no virus or viral nucleic acid was detected from nasal samples. This study identified that the seropositivity of pigs to $\mathrm{H} 1 \mathrm{~N} 1$ and $\mathrm{H} 3 \mathrm{~N} 2$ based on ELISA was significantly associated with factors such as size of farm, importation or purchase of pigs, proximity of farm to other pig farms and the presence of mammalian pets within the farm
\end{abstract}

Keyword: Malaysia; Swine influenza; Risk factors; ELISA; Mammalian pets. 